chen. Die von Landray präsentierten Subgruppen-Analysen lieferten dazu noch keine entscheidenden Hinweise. Eine interessante Wende hat hier die Entwicklung des vermeintlich wirkungslosen Dalcetrapib genommen. Denn inzwischen besteht die Hoffnung, dass Gene den Weg zu den "richtigen“ Patienten für diese Therapie weisen könnten.

Nach Genotypisierung von Teilnehmern der dal-OUTCOME-Studie hat sich gezeigt, dass zumindest eine Sub- gruppe - Patienten, die homozygot für eine Variante (rs1967309) im ADCY9Gen sind - von Dalcetrapib profitiert hatten. Wissenschaftlich beweiskräftig ist das noch nicht.

In einer großen Phase-III-Studie (dalGenE) soll deshalb bei rund 5.000 Patienten mit akutem Koronarsyndrom und entsprechendem Genotyp geprüft werden, ob der CETP-Hemmer bei dieser gezielt ausgewählten Patientengruppe von substanziellem Nutzen ist. Ob auch bei der weiteren Entwicklung von Ana- cetrapib in diese Richtung gegangen wird, bleibt abzuwarten.

Peter Overbeck

Quelle: Hot Line - Late Breaking Clinical Trials 4 "Clinical effects of anacetrapib in people with established vascular disease: Results of the Randomized EValuation of the Effects of Anacetrapib through Lipid-modification (HPS3/ TIMI55-REVEAL) trial“", ESC-Kongress, 28.8.2017 in Barcelona

The HPS3-TIMI55-REVEAL Collaborative Group. Effects of anacetrapib in patients with atherosclerotic vascular disease (REVEAL). N Engl J Med. 2017; https://doi.org/10.1056/NEJMoa1706444

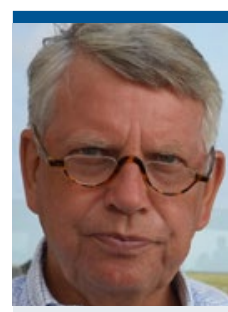

Kommentar von Prof. Gerald Klose

\title{
Chancen für einen neuen Lipidsenker?
}

Der Wirksamkeitsnachweis des CETP-Hemmers Anacetrapib hat für Aufmerksamkeit gesorgt. Das liegt vor allem daran, dass die bisherigen CETP-Hemmer an einem solchen Nachweis gescheitert sind. Hier stellt sich die Frage, was die Studien oder die Substanzen unterscheidet, welche Relevanz die Ergebnisse für die kardiovaskuläre Prävention haben und welche Perspektiven sich für eine individualisierte Lipidtherapie ergeben könnten?

Besonders fällt auf, dass sich Studiendauer und Patientenzahl in REVEAL von denen vergangenen CETP-Hemmer-Studien deutlich unterscheiden. Die Studie hat mehr als doppelt so viele Teilnehmer und eine fast doppelte Dauer als die ACCELERATE-Studie. Dies spielt angesichts der erst nach dem zweiten Jahr auseinandergehenden Kaplan-Meyer-Kurven sicher keine geringe Rolle. Die Autoren heben dies als eine der Stärken der Studie hervor, ebenso wie die Ereignisrate und die Adhärenz.

Gegenüber dem CETP-Hemmer Torcetrapib zeichnet sich Anacetrapib durch das Fehlen sog. „Off-target"-Effekte aus. Torcetrapib hatte in der ILLUMINATE-Studie durch eine Mineralkortikoid-Erhöhung zu einer Steigerung des Blutdrucks geführt. Die Lipidsenkung von Dalcetrapib könnte in dal-OUTCOME zu schwach gewesen sein, um damit Endpunkte beeinflussen zu können.

Darüber hinaus gibt es Besonderheiten in der Lipidwirkung von Anacetrapib. Hierzu gehört die kürzlich gemachte Beobachtung, dass extrem hohe HDL-C-Werte paradoxerweise negative kardiovaskuläre Effekte entfalten (Madsen CM et al. Eur Heart J. 2017). Insofern können niedrige $\mathrm{HDL}-\mathrm{C}$-Ausgangswerte wie in REVEAL entscheidend sein, ob die Therapie wirkt oder nicht.

Womöglich lässt sich der Nutzennachweis von Anacetrapib auch durch dessen Wirkung auf LDL-C und Apoprotein B erklären, die am stärksten von allen CETP-Hemmern ausfiel. Die Effekte auf Non-HDL-C wurden in den anderen CETP-Hemmer-Studien gar nicht in
Betracht gezogen. Nachdenklich macht auch die in REVEAL berichtete Lp(a)-Senkung, die einen Stellenwert haben könnte.

Die REVEAL-Autoren gehen auch auf Limitationen ein. Hierzu gehören die besonders niedrigen AusgangsLDL-C-Werte, die sich nicht ohne weiteres auf andere Situationen übertragen lassen. Eine Limitation ist auch die bislang nicht bewertbare lange Halbwertszeit der Substanz im Fettgewebe.

Hat sich mit REVEAL nun ein weiterer Lipidsenker für die kardiovaskuläre Prävention eröffnet? Diese Frage wird von Experten angesichts des moderaten Ausmaßes der Risikoreduktion zurückhaltend diskutiert. Zur Plausibilität der CETP-Hemmung trug beim ESC-Kongress das Ergebnis einer Studie nach dem Prinzip der Mendelschen Randomisierung bei. Der Wirkung von CETP-Inhibitoren entsprechende genetische CETP-Varianten gingen mit einer Lipidsenkung einher, die eine Reduktion des kardiovaskulären Risikos zur Folge hatte (Ference BA. JAMA. 2017).

Interessant ist sicher die Perspektive, die Indikationsstellung für CETP-Hemmer auf Basis der genetischen Konstellation auszurichten, wie sie sich aus Post-hocAnalysen der dal-OUTCOMES-Studie ergab (Tardif JC et al. Thromb Vasc Biol. 2017).

Gut nachvollziehbar ist das in der John Chapmans Diskussion aufgeführte mögliche Therapiefenster für Anacetrapib: Als Kombinationspartner für eine Intensivierung der lipidsenkenden Therapie bei Patienten mit hohem kardiovaskulären Risiko und niedrigem HDL-C mit komplementären Wirkungsmechanismen beispielsweise bei statinintoleranten Patienten.

\section{Prof. Dr. med. Gerald Klose}

Praxen Dres. T. Beckenbauer \& S. Maierhof, Am Markt 11, 28195 Bremen

Dres. I. van de Loo \& K. Spieker, Gerold Janssen Straße 2 A, 28359 Bremen 